\title{
Zum Stand der schweizerischen Raumplanungskartographie
}

\section{Einleitung}

\subsection{Kartographie und Raumplanung}

Das Vorhandensein von topographischen Karten und Plänen ist von grundlegender Bedeutung für die Raumplanung. Ohne Kenntnisse der topographischen Gegebenheiten des Planungsraumes und ohne Kartierungsgrundlagen lässt sich kaum eine realistische Planung durchführen. Die Karte stellt aber nicht nur eine wertvolle Grundlage für die Raumplanung dar, als zweidimensionale Darstellung der räumlichen Verbreitung von Objekten bildet sie in allen Phasen der räumlichen Planung, von der Problemerkennung bis zur Realisierung, ein wichtiges Arbeits- und Darstellungsmittel.

Erstaunlich ist nun, dass trotz dieses gemeinsamen Interessensgebietes zumindest in der Schweiz nur wenige Kontakte zwischen Raumplanung und Kartographie bestehen; dies zeigt sich beispielsweise in einem äusserst spärlichen Schrifttum zu diesem Thema. In Deutschland hat sich W. Witt ausführlich mit dem Problemkreis der Raumplanungskartographie befasst (1). Von Seiten des ORL-Institutes an der ETHZ wurde ebenfalls in letzter Zeit verschiedentlich versucht, die Raumplaner über die Leistungsmöglichkeiten der Kartographie zu orientieren sowie gegenseitige Beziehungen zwischen diesen Fachgebieten aufzuzeigen (2).

Ein Grund für die fehlenden Kontakte liegt darin, dass die Raumplanung die Möglichkeiten der Kartographie nicht voll erkannt hat. Der Raumplaner betrachtet die Kartographie häufig lediglich als Zeichentechnik und übersieht dabei, dass die Kartographie heute als Formalwissenschaft etabliert ist (3) und der Raumplanung eine Reihe von Leistungen anzubieten hat. Einen umfassenden Ueberblick über diese Leistungsmöglichkeiten bietet ein kürzlich erschienener Artikel von I. Kretschmer (4). Die Autorin sieht dabei vor allem zwei wichtige Anwendungsgebiete des Karteneinsatzes in der Raumplanung: Einerseits die Verwendung von Karten zur flächenhaften Informationsgewinnung (Analyse von Karten), anderseits die Verwendung von Karten zur flächenhaften Informationsdarbietung.

Doch nicht nur der Planer verkennt die Möglichkeiten der Kartographie, auch der Kartograph und der Kartenautor kennen oft die Bedürnisse der Planung zu wenig. Thematische Karten, die als Grundlage für die Raumplanung gedacht sind, können oftmals vom Planer gar nicht richtig verstanden werden, da sie zu wenig "in die Sprache der Raumplanung übersetzt" sind. G. Lüttig (5) schlägt deshalb vor, dass der Geowissenschafter zım Zweck der Aussagepräzisierung, aufgrund analytischer, thematischer Karten zusätzlich eigentliche Nutzungsanspruchskarten schaffen soll. Auf diese Weise sollte es möglich sein, planerische Konfliktlösungen besser vorzubereiten. Diese Forderung deckt sich mit dem bereits von verschiedenen Stellen geäusserten Wunsch, für die Raumplanung nicht nur Zustandskarten, sondern bereits synthetisierte Eignungs- und Risikenkarten zu entwickeln.

Zusammenfassend können wir feststellen, dass die Möglichkeiten der Kartographie zur Informationsgewinnung und -darbietung bei der Lösung raumplanerischer Probleme noch zu wenig ausgeschöpft werden. Auch die Raumplanung sollte sich vermehrt mit diesen Fragen auseinandersetzen. Pläne dienen ihr als wichtiges Kommunikationsmittel, sowohl mit der breiten 0effentlichkeit als auch mit den Politikern, und von einer erfolgreichen Kommunikation hängt zu einem wesentlichen Teil die Durchschlagskraft der Planung ab (6).

\subsection{Ziele des Aufsatzes}

Raumplanerische Grundlagenuntersuchungen und in diesem Zusammenhang erstellte thematische Karten enthalten für den Geographen und andere am räumlichen Geschehen interessierte Wissenschafter sehr oft eine Fülle von wertvollen Informationen. Diese Untersuchungen sind aber oft nur beschränkt verfügbar, häufig auch in Bibliotheker, nicht vorhanden und somit einer breiteren, wissenschaftlich interessierten 0effentlichkeit unbekannt.

Wir möchten mit dem vorliegenden Aufsatz versuchen, diese Informationslücke etwas zu schliessen, indem wir einen Ueberblick über diejenigen thematischen Karten vermitteln, die seit 1970 durch die kantonalen Planungsämter, entweder in Form von Aufträgen an aussenstehende Bearbeiter oder in eigener Regie, erstellt wurden. Wir beschränken uns dabei auf eine Orientierung über die Karteninhalte und verzichten auf eine Beurteilung der Karten aus kartographischer Sicht.

Dr. Heinz Trachsler und PD Dr. Hans Elsasser, ORL-Institut ETH-Hönggerberg, 8093 Zürich 
Um eine derartige Uebersicht zu erstellen, führten wir im Sommer 1977 eine Umfrage bei den kantonalen Planungsstellen durch. Wir möchten an dieser Stelle diesen Aemtern für ihre bereitwillig erteilten und teilweise sehr umfassenden Antworten bestens danken. Die gestellten Fragen lauteten folgendermassen:

- Welche thematischen Karten sind seit 1970 durch Ihre Planungsstelle, bzw. in Ihrem Auftrag erarbeitet worden (Titel der Karte, Massstab, Erscheinungsjahr, Bearbeiter)?

- Existieren in Ihrem Kanton Richtlinien für die kartographische Gestaltung von Richt- und Zonenplänen, oder ist vorgesehen, solche zu erlassen?

- Wieviele Mitarbeiter sind in Ihrer Amtsstelle mit der graphischen Gestaltung von Karten und Plänen beschäftigt? Handelt es sich dabei um ausgebildete Kartographen, Zeichner oder Graphiker? Vergeben Sie Ihre kartographischen Arbeiten an auswärtige Firmen oder freiberuflich tätige Kartographen?

Bei der Beurteilung der Ergebnisse darf nicht übersehen werden, dass deren Aussagekraft beschränkt ist, und dass sie keinesfalls ein vollständiges Bild über die kartographischen Tätigkeiten auf dem Gebiet der schweizerischen Raumplanung vermitteln. Kommunale Amtsstellen, Regionalplanungsgruppen und private Planungsbüros wurden bei der Umfrage nicht berücksichtigt. Ueber die kartographischen Aktivitäten des Delegierten für Raumplanung gibt der Artikel von $H$. Leuzinger in diesem Heft Auskunft. Ferner ist zu beachten, dass bei der Auswertung der Umfrage nur thematische Karten, d.h. Zustandskarten sowie eigentliche Planungsgrundlagenkarten (z.B. Eignungs-, Risiko-, Prognose-, Bedarfskarten usw.), hingegen keine kantonalen Leitbilder und Richtpläne berücksichtigt wurden. Zudem sollte es sich bei den in der Uebersicht erwähnten Karten um eigenständige Publikationen und nicht um kleinmassstäbige Abbildungen im Rahmen von Berichten und Gutachten handeln.

\section{Ergebnisse der Umfrage}

\subsection{Planungsatlanten}

Die Kantone Bern und Freiburg verfügen über. eigene Planungsatlanten (7). Nach Witt (8) kann zwischen Planungsgrundlagen- oder Strukturatlanten einerseits und Planungsatlanten i.e.S. anderseits unterschieden werden. Die Strukturatlanten bestehen dabei vor allem aus analytischen Zustandskarten, welche über die räumlichen Strukturen des Planungsraumes orientieren. Der Planungsatlas i.e.S. hingegen setzt sich in erster Linie aus Karten zusammen, die direkt auf raumplanerische Problemstellungen und Konzepte ausgerichtet sind. Unter Berücksichtigung dieser Terminologie sind die beiden Atlaswerke eher den Strukturatlanten zuzuordnen. Dasselbe gilt für die vom Stadtplanungsbureau BaselStadt 1975 herausgegebene Bestandesaufnahme im Hektarraster. Das Planungsamt des Kantons Waadt veröffentlichte 1974 den umfangreichen 'Atlas statistique agricole vaudois'. Auf gesamtschweizerischer Ebene existiert kein Planungsatlas (9). Der Atlas der Schweiz übernimmt für gewisse beschränkte, vor allem nationale Bereiche auch die Aufgaben eines schweizerischen Planungsatlas'.

\subsection{Thematische Einzelkarten}

In der folgenden Zusammenstellung haben wir die von den einzelnen kantonalen Planungsstellen seit 1970 für die Raumplanung erarbeiteten Karten nach bestimmten Themenkreisen geordnet:

Klima:

In den Kantonen Bern und Waadt wurden in Zusammenarbeit mit den $\mathrm{Planungsämtern} \mathrm{Kl}$ imauntersuchungen durchgeführt, deren Resultate teilweise in Form von Karten vorliegen:

- Beiträge zum Klima des Kantons Bern, 1 : 200'000 und 1: 300'000, 1977 (Geographisches Institut der Universität Bern)

- Niveaux thermiques du canton de Vaud, 1 : $100^{\prime} 000,1968$ (K.F. Schreiber)

- Dangers de gel tardif printanier dans le canton de Vaud, 1: 100'000, 1971 (K.F. Schreiber)

- Série de cartes mésoclimatiques du canton de Vaud, 1 : 200'000, 1972 (B. Primault)

Geologie u.ä.:

Unter dem Stichwort 'Geologie u.ä.' können die nachfolgenden Karten zusammengefasst werden:

- Hydrogeologische Karte des Kantons Nidwalden, 1 : 25'000, 1973 (L. Wyssling)

- Baugrundzonenkarte Region Basel, 1 : 25'000, 1972 (G. Wiener)

- Ausbeutungszonen mineralischer Rohstoffe Region Basel, 1 : 25'000, 1972 (G. Wiener)

- Karte der Kiesvorkommen des Kantons Thurgau, 1 : 50'000, 1973 (Amt für Raumplanung des Kantons Thurgau)

- Carte lithologique du canton de Vaud, 1 : $25^{\prime} 000,1968$ (M. Burri)

- Cartes des sources du canton de Vaud, 1 : 25'000, 1963 - 1977 (E. Badoux, J. Mautner)

Gefahrengebiete:

Gefahren- oder Risikenkarten wurden in den Kantonen Freiburg und Waadt erarbeitet:

- Vorläufige Uebersichtskarte der Rutschgebiete im Kanton Freiburg, 1 : 50'000, 1976 (Kantonale Kommission für die Erstellung eines Lawinenkatasters u.a.)

- Uebersichtskarte der Lawinengebiete des Kantons Freiburg, 1 : 50'000, 1976 (Kantonale Kommission für die Erstellung eines Lawinenkatasters)

- Cadastre des avalanches du canton de Vaud, 1 : 25'000, 1968 (J.-P. Bezençon)

- Cartes de la stabilité des sols du canton de Vaud, 1 : 25'000, in Bearbeitung (M. Gabus)

- Carte des zones soumises à des dangers naturels du canton de Vaud, 1 : 100'000 (Service de 1 'aménagement du territoire)

\section{Schutzgebiete:}

Im Zusammenhang mit dem 1972 erlassenen Bundesbeschluss über dringliche Massnahmen auf dem 
Gebiete der Raumplanung, wurden die Kantone verpflichtet, provisorische Schutzgebiete auszuscheiden. In allen Kantonen bestehen deshalb Karten im Massstab 1: 25'000, auf denen diese Schutzgebiete eingetragen sind. In einzelnen Kantonen wurden aber noch zusätzliche Schutzgebietskarten erstellt:

- Karte der Gewässerschutzbereiche des Kantons Nidwalden, 1 : 25'000, 1974 (L. Wyssling)

- Naturschutzgutachten N.J-Schweiz, naturschutzwürdige Gebiete und Objekte, 1 : 25'000 und 1 : 100'000 (Schweizerischer Bund für Naturschutz, K. Ewald)

- Inventar der Naturschutzgebiete des Kantons Thurgau, 1 : 25'000, 1976/77 (Schweizerischer Bund für Naturschutz)

- Cartes de protection des eaux du canton de Vaud, 1 : 25'000, in Bearbeitung ( $E$. Bosset)

- Carte des sites méritant d'être protégés du canton de Vaud, 1 : 100'000, 1972 (J.-P.Reitz)

Landwirtschaft:

In zahlreichen Kantonen wurden im Auftrag der Raumplanung landwirtschaftliche Eignungskarten erarbeitet. Diese Aufgabe wurde oft der Arbeitsgemeinschaft beratender Agronomen (AGBA) in Ebikon übertragen:

- Landwirtschaftliche Eignung Kanton Zürich, 1 : 25'000, 1976 (Landwirtschaftsamt)

- Landwirtschaftliche Eignungskarte für den Kanton Bern, 1 : 25'000, 1972 (Planungsamt)

- Landwirtschaftliche Eignungskarte Kanton Luzern, 1 : 25'000, 1970 (AGBA)

- Landwirtschaftliche Eignungskarte zum Entwicklungskonzept Uri, 1 : 25'000, $1974-77$ (Institut d'économie rurale EPFZ, Th. Abt)

- Landwirtschaftliche Eignungskarte Kanton Nidwalden, 1 : 25'000, 1976 (AGBA)

- Landwirtschaftliche Eignungskarte Kanton Freiburg, in Bearbeitung

- Landwirtschaftsgutachten Basel-Landschaft, Inventar-, Eignungs- und Planungskarte, $1: 25^{\prime} 000,1970$ (AGBA)

- Landwirtschaftliche Eignungskarte des st.gallischen Berggebietes, 1 : 25'000, 1975 (AGBA)

- Carte écologique-physiographique des sols du canton de Vaud, 1 : 100'000, 1971 (R. Haeber1i)

- Carte du potentiel naturel des surfaces agricoles du canton de Vaud, 1 : 100'000, 1971 (K.F. Schreiber, R. Haeberli)

- Carte du potentiel fourrager des sols agricoles du canton de Vaud, 1: 50'000, 1976

(J.-D. Galland)

Siedlung, Bauzonen, Baugebiete:

- Zweithäuser und Schrebergärten Basel-Stadt und Basel-Landschaft, Inventarkarte, 1 : 25'000, 1970 (P. Suter)

- Nettosiedlungsfläche Basel-Stadt und BaselLandschaft, 1 : 25'000, 1975 (Regionalplanung beider Basel)

- Flächenstatistik Kanton Graubünden, Nachführung der Bauzonen und Baugebiete (Planungsstelle)

- Baugebiet/Kulturland Kanton Aargau, 1 : 25'000 und $1: 300^{\prime} 000,1977$ (Planungsamt)

- Zusammenstellung der bestehenden Bauzonen des Kantons Thurgau, 1 : 10'000, 1976 (Arbeitsgemeinschaft Fingerhuth/Keller/de Vries)

- Carte du réseau urbain du canton de Vaud, 1 : 200'000, in Bearbeitung (Service de 1 'aménagement du territoire)

- Carte de l'évolution des aires d'influence des centres du canton de Vaud 1950, 1960, 1970, 1 : 100'000, 1965 - 1974 (Service de l'amēnagement du territoire)

- Carte des propriétés publiques du canton de Vaud, 1 : 25'000, 1965 (Service des forêts)

- Carte des isochrones à partir des centres régionaux du canton de Vaud, 1 : 100'000 (Service de l'aménagement du territoire)

- Série de cartes au 1: 10'000 de 1'agglomeration de Genève: densité de la population, des emplois, des êlèves; plan des contraintes; localisation des équipements publics; les équipements d'infrastructure; le réseau routier; les disponibilités en terrain

Diverses:

- Bevölkerungsverteilung im Kanton Zürich, 1 : $100^{\prime} 000,1975$ (Amt für Raumplanung und Statistisches Amt)

- Trink- und Brauchwasserversorgung im Kanton Luzern, 1 : 100'000, 1971

- Wasserversorgungsatlas für den Kanton Nidwalden, 1 : 25'000, 1972 (Technisches Zeichenbüro Ebikon)

- Elektrizitätsversorgung Region Basel, 1 : 25'000, 1970 (Regionalplanung Baselland)

- Einkaufszentren in der Nordwestschweiz, 1 : 200'000, 1975 (Regionalplanung beider Basel)

- Landschaftstypisierung Kanton Aargau, 1 : $25^{\prime} 000,1976 / 77$ (Planungsamt)

- Fauna des Kantons Thurgau, 1: 25'000, 1977 (H. Pfister)

- Carte des localisations préférentielles des entreprises industrielles du canton de Vaud, 1 : 200'000 (Service de 1'aménagement du territoire)

Carte des équipements et potentialitẽs touristiques pour les Alpes, les Préalpes et la Vallée de Joux (Service de l'aménagement du territoire)

- Carte du domain skiable du canton de Vaud, 1 : 10'000, 1 : 25'000, 1968 - 1977 (Sociēté générale pour l'industrie)

\subsection{Richtlinien}

Bereits 1945 beschäftigte sich die Schweizerische Vereinigung für Landesplanung mit der kartographischen Darstellung von Plänen und mit der Ausarbeitung einer einheitlichen und umfassenden Legende für Nutzungskarten und -pläne: "Die Ausarbeitung einer solchen Legendur erscheint zunächst eine einfache Aufgabe zu sein. Auf der einen Seite gibt es eine endliche Zahl von Inhalten, welche dargestellt werden sollen; auf der andern Seite eine beschränkte Anzahl von Darstellungsmitteln, welche eine solche Darstellung tatsächlich ermöglichen. Nun - so möchte man glauben - genügt es, den Inhalten die 
Mittel zuzuordnen, und die Aufgabe ist gelöst. Theoretisch ist sie es auch. Sobald die Aufgabe aber praktisch angepackt wurde, erwies es sich als notwendig, zuvor Anwendungsbereich und Legendur festzulegen, sodann die brauchbaren Darstellungsmittel auszuscheiden und schliesslich die wirre Fülle von möglichen Darstellungsinhalten zu ordnen und Inhalte, die begrifflich nicht eindeutig sind, soweit als möglich zu verdeutlichen" (10). Mit diesen komplexen Fragen von Darstellungsrichtlinien in der Raumplanung beschäftigte man sich aber nicht nur in der Schweiz sondern auch im Ausland (11).

1966 wurden im Rahmen der Provisorischen Richtlinien zur Orts-, Regional- und Landesplanung Richtlinien zur graphischen Gestaltung von Zonenplänen (Blatt 511401/2/3; Bearbeiter: E. Spiess und $E$. Winkler) herausgegeben. Diese Sammlung der ORL-Richtlinien enthält ferner weitere Musterlegenden:

- Blatt 512542: Musterlegende zur Karte der geeigneten Landwirtschaftsgebiete und zur Karte der natürlichen Eignung für die Landwirtschaft

- Blatt 512621: Musterlegende und Darstellungsmuster für den Schutzplan

- Blatt 512622: Musterlegende zur Karte der schützenswerten Naturobjekte und Landschaften

- Blatt 516022: Richtlinien für die kartographische Darstellung der Grundwasserverhältnisse in Lockergesteinen

Ein abgerundetes Richtlinienwerk der graphischen Darstellung von Plänen, dessen wesentliche Inhalte ein umfassendes graphisches Plansystem, eine Mustersammlung von Planfarben und Signaturen und eine Sammlung von Planbeispielen bzw. Musterplänen sein müsste, fehlt leider heute (noch) in der Schweiz. Vorarbeiten dazu wurden von E. Winkler geleistet (12). Es ist zu hoffen, dass man sich nach dem Erlass des eidgenössischen Raumplanungsgesetzes erneut und vermehrt auch mit dem Problemkreis der Richtlinien für die kartographische Darstellung der kantonalen Richtpläne beschäftigen wird. Für eine Koordination der kantonalen Richtplanungen ist eine einheitliche oder zumindest vergleichbare Darstellung dieser Pläne eine wichtige Voraussetzung Es bleibt zu hoffen, dass bei der Erarbeitung künftiger Darstellungsnormen neben dem Planungsfachmann auch der Kartograph als Berater beigezogen wird.

Bereits heute verfügen aber einzelne Kantone über Richtlinien für die graphische Gestaltung von Richt- und Zonenplänen:

Zürich Darstellungsrichtlinien für die Richt planung, für die Nutzungsplanung in Bearbeitung.

Bern Richtlinien für die kartographische Gestaltung und den Inhalt von Richtund Zonenplänen.

Luzern Entwurf von Weisungen und Erläuterungen zum Vorgehen für die Darstellung von Ortsplanungen.

Solothurn Einzelne Richtlinien über Inhalt und Darstellung der Zonen-, Strassen- und Baulinienpläne der Gemeinden; Richtlinien über Inhalt und Darstellung der Richtpläne.

Basel- Darstellungsnormen für die Orts-

Landschaft planung.

Graubünden Richtlinien für die kartographische Gestaltung von Richt- und Zonenplänen.

Thurgau Darstellungsrichtlinien für Zonenpläne.

Vaud

Wallis Légende type pour plans des zones.

Genève Richtlinien für die kartographische Gestaltung von Richtplänen. Richtlinien für die Legenden der Richt- und Zonenpläne vorgesehen.

\subsection{Kartographen in der Raumplanung}

In den kantonalen Planungsämtern sind rund 40 Mitarbeiter mit der graphischen Gestaltung von Karten und Plänen betraut. Sie setzen sich aus den folgenden Berufsgruppen zusammen: Bauzeichner (58\%), Planungszeichner (13\%), Graphiker (13\%), Kartographen (8\%), angelernte Hilfskräfte $(8 \%)$. Einzelne Kantone vergeben auch Aufträge an freierwerbende Kartographen.

$\mathrm{Zu}$ beachten ist die geringe Zahl von Kartographen. Dieses Ergebnis deckt sich mit der bereits 1972 gemachten Feststellung der Schweizerischen Gesellschaft für Kartographie, dass der Beruf des Kartographen in der Raumplanung nahezu unbekannt sei (13). Bereits seit mehreren Jahren existiert zudem der Beruf des Planungszeichners, mit einer speziell auf die Bedürnisse der Raumplanung ausgerichteten Ausbildung. In Zukunft dürten sich die Einsatzmöglichkeiten des Kartographen vor allem auf die Herstellung von gesamtschweizerischen und eventuell kantonalen Planungsgrundlagen- und Planungskarten beschränken. Die graphischen Gestaltungen im Rahmen der Ortsund Regionalplanungen dürten jedoch vermehrt an die Planungszeichner ubergehen. Es ist zu hoffen, dass Erfahrungen und Erkenntnisse aus der Kartographie sowohl im graphischen als auch im reproduktionstechnischen Bereich optimal verwertet werden.

\section{Schlussfolgerungen}

Die Zusammenstellung der durch die Kantone veröffentlichten Planungskarten zeigt, dass auch in der Schweiz auf dem Gebiet der Raumplanungskartographie in den letzten Jahren eine beachtliche Aktivität eingesetzt hat. Erfreulich dabei ist, dass der Wert der thematischen Karte als Kommunikationsmittel zwischen Geowissenschaftern, Planern, Politikern und der Oeffentlichkeit immer mehr erkannt worden ist. Es muss allerdings festgestellt werden, dass diese Karten zu einem geringen Teil von Kartographen erstellt wurden.

Positiv ist die Veröffentlichung von Planungsatlanten zu werten, vermitteln sie doch dem Planer in übersichtlicher Form eine Fülle von Informationen über den Planungsraum. Allerdings ist, bedingt durch den hohen zeitlichen und finanziellen Aufwand, den die Herstellung derartiger At- 
lanten mit sich bringt, in nächster Zeit kaum mit der Veröffentlichung neuer Atlaswerke zu rechnen. Auch das Projekt 'Raumplanungsatlas der Schweiz' wird am ORL-Institut der ETH vorläufig nicht mehr weiter verfolgt. Es hat sich überdies gezeigt, dass ein Atlas in der traditionellen Buchform, oder aufgeteilt in einzelne Lieferungen, die durch eine rollende $\mathrm{Pla}-$ nung entstehenden Bedürfnisse nach Aktualität nicht zu befriedigen vermag. Die Gefahr des Veralterns ist bei derartigen Kartenwerken sehr gross. Eine geeignete Lösung bildet hingegen die Veröffentlichung von Einzelkarten, welche durch einen erläuternden und interpretierenden Begleittext ergänzt sind. Gute Beispiele dafür bilden die früher vom ORL-Institut herausgegebenen Karten ( $K$ l imaeignung für Landwirtschaft, Klimaeignung für Siedlung und Erholung, lawinengefährdete Gebiete, geotechnische Risiken) und die landwirtschaftliche Bodeneignungskarte der Schweiz, welche vom Delegierten für Raumplanung herausgegeben wird. Nach diesem System sind beispielsweise auch die Planungsatlanten von Nordrhein-Westfalen und Hamburg aufgebaut.

Es wäre sicher zu begrüssen, wenn auch in der Schweiz die Möglichkeiten geschaffen würden, ein auf dieser Form der Publikation aufbauendes Kartenwerk zur Raumplanung zu veröffentlichen. Vielleicht ein erster Schritt in dieser Richtung könnte die Erfassung aller raumplanerisch relevanter Karten der Schweiz darstellen. Dies könnte z.B. erfolgen, indem die in diesem Aufsatz dargestellte Kartenübersicht in Zukunft laufend ergänzt und nachgeführt würde. Ein solches Vorhaben dürfte sicher sowohl für die Raumplanung, für die Geowissenschaften als auch für die Kartographie von Interesse sein und die Zusammenarbeit zwischen diesen Fachbereichen fördern.

\section{Anmerkungen:}

(1) Vgl. dazu z.B. die folgenden Publikationen von Witt:

- Thematische Kartographie und Raumforschung, Akademie für Raumforschung und Landesplanung (Hrsg.), 25 Jahre Raumforschung in Deutschland, Bremen 1960

- Die kartographische Bestandesaufnahme in der Raumforschung und Landesplanung, Entwicklung und Problematik, Informationen $\mathrm{Nr} .19 / 20,1960$

- Komplexe Themakartographie in der Landesplanung, Kartographische Nachrichten $\mathrm{Nr}$. 5,1961

- Planungskartographie, Untersuchungen zur thematischen Kartographie, Veröffentlichungen der Akademie für Raumforschung und Landesplanung, Forschungs- und Sitzungsberichte $\mathrm{Nr}$. 51, Hannover 1969
(2) Trachsler Heinz, Kartographie und Raumplanung, ORL-DISP Nr. 37 (1975)

Bernath Hans Jakob und Elsasser Hans, Kartographische Darstellungsprobleme in der Raumplanung, ORL-DISP Nr. 40 (1976)

Elsasser Hans, Gruppenbildung und Wahl der Bezugsflächen bei der kartographischen Darstellung von raumplanerischen Daten, KTBLArbeitsblatt $\mathrm{Nr}$. 3031, Darmstadt 1976 Gresch Peter, Raster als Darstellungsmittel für die Raumplanung, ORL-DISP Nr. 44 (1977)

(3) Kretschmer Ingrid, Was kann die Kartographie für die Umweltplanung leisten?, Kartographische Nachrichten $\mathrm{Nr} .1,1977$, S. 10

(4) Kretschmer Ingrid, a.a.0.

(5) Lüttig Gerd, Die Rolle der geowissenschaftlichen Kartographie in der vorausschauenden Umweltforschung, Kartographische Nachrichten $\mathrm{Nr}$. 3, 1977

(6) Kretschmer Ingrid, a.a.0., S.15

(7) Planungsamt und Geographisches Institut der Universität Bern, Abteilung für angewandte Geographie, Planungsatlas Kanton Bern I - III, 1969 - 1973 Amt für Raumplanung Jes Kantons Freiburg, Kantonaler Planungsatlas, Freiburg 1973

( 8) Witt Werner, Planungsatlanten, Akademie für Raumforschung und Landesplanung (Hrsg.), Handwörterbuch der Raumforschung und Raumordnung, 2. Auflage, Hannover 1970 Witt Werner, Regional- und Planungsatlanten, Allgemeine Vermessungsnachrichten $\mathrm{Nr}$. 4, 1972

(9) Winkler Ernst, Ein Raumplanungsatlas der Schweiz, ORL-DISP Nr. 28, (1973) Winkler Ernst, Ein Raumplanungsatlas der Schweiz, Vermessung, Photogrammetrie, Kulturtechnik Nr. 1, 1975 (Festschrift Ed. Imhof)

(10) Aregger Hans, Die Möglichkeiten in der Darstellung von Plänen, Heftenreihe der Schweizerischen Vereinigung für Landesplanung $\mathrm{Nr} .1,1945$

Vgl. ferner Hoechel A., Essai d'un système graphique pour traduire l'utilisation de l'espace dans les plans d'urbanisme, UIA commission de travail 5 urbanisme, Separatdruck aus Bulletin technique de $1 \mathrm{a}$ Suisse romande No. 14, 1953

(11) Als Beispiele seien erwähnt: Oesterreichische Gesellschaft zur Förderung von Landesforschung und Landesplanung, Planzeichen für rechtswirksame Flächenwidmungspläne, Wien 1961 Planzeichen für Raumordnungs- und Entwicklungspläne, Schriften der Landesplanung Niedersachsen, Sonderveröffentlichung $\mathrm{Nr}$. 2, Hannover 1968

(12) Winkler Ernst, Graphische Darstellung (Ausarbeitung von Richtlinien und Darstellungsnormen, 1. Etappe), Schlussbericht zum FAPAuftrag 5007, ORL-Institut ETHZ, Zürich 1973 (vervielfältigt)

(13) Schweizerische Gesellschaft für Kartographie (Exposé des Vorstandes), Die Situation der schweizerischen Kartographie heute und in der Zukunft, 1972 